ÉGYPTE monde arabe

\section{Égypte/Monde arabe}

15 | 2017

Migrations vues d'Égypte - mobilisations et politiques

\title{
Réfugiés syriens en Égypte : Secteur associatif et reconfiguration étatique (2011-2013)
}

\section{Maaï Youssef}

\section{Q OpenEdition}

1 Journals

Édition électronique

URL : https://journals.openedition.org/ema/3669

DOI : 10.4000/ema.3669

ISSN : 2090-7273

Éditeur

CEDEJ - Centre d'études et de documentation économiques juridiques et sociales

Édition imprimée

Date de publication : 5 juin 2017

Pagination : 71-94

ISBN : 2-905838-93-0

ISSN : 1110-5097

\section{Référence électronique}

Maaï Youssef, « Réfugiés syriens en Égypte : Secteur associatif et reconfiguration étatique

(2011-2013) », Égypte/Monde arabe [En ligne], 15 | 2017, mis en ligne le 05 juin 2019, consulté le 07

juillet 2022. URL : http://journals.openedition.org/ema/3669 ; DOI : https://doi.org/10.4000/ema.3669 


\section{RÉFUGIÉS SYRIENS EN ÉGYPTE:}

SECTEUR ASSOCIATIF

ET RECONFIGURATION ÉTATIQUE (2011-2013)

RÉSUMÉ

Depuis mars 2011, l'Égypte est confrontée à la vague migratoire la plus importante de son histoire: celle des réfugiés syriens fuyant le régime de Bachar al-Assad. Les discours politiques du président Mohamed Morsi, entre juin 2012 et juillet 2013, plaident en faveur d'une prise en charge de ces derniers par l'État égyptien, en raison d'un soutien affiché au mouvement contestataire syrien. Dans les faits, on voit davantage se dessiner une politique publique par «à coups » et par «ébauche» qui est en effet une succession de discours officiels et de décrets ponctuels. En pratique, l'accueil des réfugiés syriens n'est pas administré, organisé et financé par des appareils étatiques. Le secteur associatif émergent et la base militante et sociale des Frères musulmans, déjà en place durant les années de semi-clandestinité de I'organisation, jouent un rôle majeur face à l'inaction des services publics. Cet article tente de comprendre les mécanismes de coproduction d'une politique de décharge, menée entre ces différents acteurs. Ceci éclaire sur la compétition autour de l'exercice du pouvoir en cours à la tête de l'État.

\section{INTRODUCTION}

Dès 2011 en Syrie, la répression sanglante du mouvement révolutionnaire par le régime de Bachar al-Assad, conduit des milliers de Syriens à quitter leur foyer, pour une autre région de la Syrie ou pour un autre pays. Les pays d'accueil frontaliers (Liban, Turquie et Jordanie notamment) sont les exemples les plus connus d'arrivées importantes de réfugiés syriens. L'Égypte est un cas 
moins représenté dans les médias, ainsi que dans la littérature en sciences humaines et sociales. Très vite pourtant, le pays est confronté au mouvement migratoire le plus important de son histoire ${ }^{1}$. Certains réfugiés choisissent en effet l'Égypte en raison, entre autres, des facilités d'accès au territoire pour les Syriens, liés au passé politique et historique commun entre les deux pays (la République arabe unie notamment, 1958-1961). Les citoyens syriens peuvent ainsi se rendre en Égypte sans demande de visa préalable. Entre 2011 et 2013, plus de 130000 réfugiés syriens sont enregistrés par l'Agence des Nations Unies pour les réfugiés $(\mathrm{HCR})^{2}$. Les associations estiment à 500000 le nombre de Syriens véritablement présents sur le territoire égyptien, tandis que les autorités égyptiennes parlent de 300000 Syriens. Ce décalage s'explique en partie par le fait qu'un nombre important de Syriens refuse d'être enregistrés en tant que réfugiés. Les raisons sont multiples, comme par exemple les craintes de représailles une fois de retour en Syrie ${ }^{3}$.

Dans la capitale cairote mais pas uniquement, le contexte d'urgence humanitaire dû aux situations de ruptures sociale, économique et politique brutales, les défis quotidiens liés à la migration (scolarisation, emploi, santé), la volonté de poursuivre la mobilisation politique contre le régime syrien en exil, conduisent à l'émergence d'initiatives collectives. Ces dernières empruntent des formes diverses. Ainsi, de 2011 à 2013, période encore marquée par la dynamique révolutionnaire en Égypte, un foisonnement d'initiatives locales et transnationales apparaît. A partir de juin 2012, le président islamiste Mohamed Morsi, issu des rangs des Frères musulmans, manifeste son soutien au mouvement révolutionnaire syrien. Il encourage ainsi une politique d'accueil favorable aux réfugiés syriens en Égypte. Emerge, dans les discours et représentations des acteurs syriens et égyptiens, I'idée d'une société civile syrienne en Égypte.

1. ZOHRY, Ayman, "The Place of Egypt in the Regional Migration System as a Receiving Country», Revue européenne des migrations internationales, vol. 19, n³, 2003, mis en ligne le 13 octobre 2004, consulté le 1 juin 2016. URL : http://remi. revues.org/2664.

2. ChiffresofficielsduHCR,URL:http://reporting.unhcr.org/node/2540?y=2014\#year, consulté le 19 janvier 2017.

3. Ces dernières peuvent être nourries par des menaces et pressions répétées de la part de l'ambassade de Syrie en Égypte sur ses ressortissants. Plusieurs entretiens font état de ces faits, I'ambassade de Syrie en Égypte, pro-régime de Bashar al- Assad, refuse de renouveler les passeports des ressortissants syriens présents sur le territoire égyptien, leur imposant une clandestinité, vécue par les migrants forcés syriens comme une "punition » et le gage de représailles dans le cas d'un retour en Syrie sous le même régime. Des cas de délation de la part de membres de l'ambassade aux autorités égyptiennes, sont aussi relatés dans les entretiens. 
J'explorerai dans cet article un aspect de ce que recouvre cette notion endogène de société civile syrienne: le secteur associatif de l'accompagnement aux réfugiés syriens, visant à améliorer les conditions de leur intégration en Égypte. Dans les grandes villes notamment (Alexandrie, Le Caire, Six Octobre), un tissu d'institutions - associatives, artistiques, intellectuelles, religieuses, communautaires, professionnelles ou encore politiques - se développe. La dimension associative de la société civile syrienne constitue pour le chercheur, et telle que le préconisait Michel Camau, une catégorie de l'ac$t_{i o n}{ }^{4}$ pertinente car investie par les acteurs au cœur de cette recherche. Le secteur associatif est principalement organisé autour de la ville de Six Octobre. Située en périphérie de la capitale, à la limite avec le désert, cette ville nouvelle est née en 1979 avec le but de désengorger Le Caire. Elle accueille plus de la moitié des Syriens présents sur le territoire égyptien, soit entre soixante mille et cent mille Syriens au regard des différentes estimations. Les acteurs impliqués dans ce secteur associatif sont principalement des Syriens, issus de la classe moyenne, voire de la petite bourgeoisie, anciens professeurs, étudiants, avocats, médecins et infirmiers, propriétaires de petits commerces, employés d'entreprise syrienne, travaillant par exemple dans l'import-export.

Deux types de revendication et de motivation se croisent dans les discours des acteurs. Tout d'abord, on note la volonté politique manifeste de poursuivre le mouvement contestataire syrien en exil. Les actions et mobilisations peuvent avoir un caractère social, dans leurs formes institutionnelles et organisationnelles (ouvertures de centres communautaires, distributions de biens de première nécessité par exemple), mais il n'en demeure pas moins qu'elles sont pensées par les acteurs comme des formes de résistance contre un régime syrien, qui condamne une partie de sa population à quitter malgré elle son pays. En parallèle, certaines initiatives sont présentées comme uniquement caritatives et humanitaires. Dans les discours de leurs fondateurs, la dimension apolitique est motrice, leurs actions se veulent impulsées par la nécessité d'accompagner économiquement et socialement les Syriens dans les routines complexes de la migration forcée, tout en restant en dehors du conflit politique syrien. La frontière est bien sûr poreuse entre ces différents aspects de I'action sociale, questionnant comment et pourquoi se mettent en place les pratiques et stratégies des acteurs mobilisés au sein de ce secteur associatif en devenir. Alors que les appareils étatiques sont particulièrement polarisés en Égypte entre Frères musulmans et autres forces politiques, liées pour partie à I'ancien régime, quel rapport entretiennent les acteurs mobilisés au sein du

4. CAMAU, Michel, "Sociétés civiles «réelles » et téléologie de la démocratisation », Revue internationale de politique comparée, 2, p. 213-232. 
secteur associatif avec le politique? Que disent leurs pratiques et leurs stratégies à propos des formes de gestion étatique, multiples et parfois contradictoires, d'un foisonnement important d'initiatives sociales et politiques?

Cet article s'inscrit dans la lignée du travail sur la société civile et le secteur associatif en Égypte de Sarah Ben Nefissa ${ }^{5}$. Elle explique que le secteur associatif égyptien a depuis longtemps constitué «une sorte de démembrement de l'administration chargé de pallier le désengagement social de l'État » ${ }^{6}$, participant d'une stratégie de dépolitisation des institutions, notamment locales, de la part du régime politique égyptien. Et elle poursuit ainsi :

Il est même possible de poser l'hypothèse que les associations égyptiennes ont constitué et constituent jusqu'à aujourd'hui des sortes de 'municipalités par défaut' qui présentent l'avantage, pour l'État égyptien, d'éviter la 'politisation' de la chose publique locale et de prolonger l'interdit du politique qui caractérise ses structures administratives locales.

Ces éléments font vivement écho à la situation observée, dans la ville du Six Octobre en particulier, après 2011. Le développement des formes d'accueil et d'intégration des Syriens, l'émergence du secteur associatif évoqué, le décalage entre les discours politiques et les pratiques locales, paraissent constituer des objets d'analyse permettant de prolonger les questionnements de l'auteure. L'hypothèse ici soutenue est donc que les acteurs mobilisés s'organisent en réaction à ce contexte décrit par Sarah Ben Nefissa et à ses mécanismes, et qu'ils s'inscrivent aussi dans une forme implicite et souvent silencieuse de coproduction d'une politique de décharge ${ }^{7}$, visant à organiser I'accueil et la prise en charge des réfugiés syriens. En effet, l'État égyptien, incarné à partir de 2012 par Mohamed Morsi, donne à voir des orientations politiques volontaristes. En revanche, sur le terrain la mise en œuvre de ces dernières par les relais étatiques est quasi-inexistante. Il est alors nécessaire de rendre compte: avec qui et comment se fait cette politique de décharge, qui vient pallier le non-engagement de l'État sur cette question sociale de

5. BEN NEFISSA, Sarah, "Les dynamiques sociales et politiques paradoxales de la promotion de la société civile en Égypte», in Anna Bozzo et al., Les sociétés civiles dans le monde musulman, La Découverte, 2011, p. 325-340.

6. Op. cit., p. 330.

7. HIBOU, Béatrice, La privatisation des États, Khartala, Paris. 
l'accompagnement des réfugiés syriens? En prêtant attention aux «pratiques par lesquelles s'énonce le dissentiment ${ }^{8}{ }^{8}$, cet article s'inspire du courant du politique par le bas, initié en études africaines par Jean-François Bayart ${ }^{9}$.

Dans cet article, on s'attarde sur deux exemples associatifs dans la ville de Six Octobre, rebaptisée "La Petite Syrie». La première association s'appelle Zaytoun, il s'agit d'une initiative syrienne, portée notamment par Khaïs, opposant syrien exilé au Caire. Son cas permet de comprendre les facteurs ayant permis au secteur associatif d'émerger et de se développer, avec deux idées centrales: celle du relâchement de l'appareil sécuritaire entre 2011 et 2013, et d'une instabilité institutionnelle au sein de l'État, entraînant une compétition pour le pouvoir dans les organes étatiques. La seconde association étudiée se nomme Unique, il s'agit d'une association locale égyptienne, portée par des fondateurs majoritairement égyptiens et une équipe de bénévoles syriens. Le cas d'Unique permet d'explorer la thèse $d^{\prime}$ une politique de décharge, qui serait impulsée par les Frères musulmans eux-mêmes pour pallier leur incapacité à prendre les rênes du pouvoir, au cours de la présidence de Mohamed Morsi. Les données présentées dans cet article ont été recueillies dans le cadre d'enquêtes de terrain, menées entre 2013 et 2014, en Égypte et en Turquie. Soixante entretiens semi-directifs et récits de vie ont été conduits au cours de cette période, ainsi que des observations au Caire et dans la ville du Six Octobre.

\section{RÉFUGIÉS SYRIENS:}

TRANSFORMER L'ENGAGEMENT POLITIQUE EN ACTION SOCIALE-

\section{Rester mobilisés en exil}

Le secteur associatif de l'aide aux réfugiés syriens émerge dans le contexte d'instabilité politique lié à la chute du régime d'Hosni Moubarak. Des acteurs syriens mettent à profit, bien souvent sans le savoir, les largesses permises par I'atmosphère politique et le soutien de Mohamed Morsi au mouvement révolutionnaire syrien, pour se mobiliser depuis l'Égypte. Ils mettent en place leur propre organisation de l'aide et de l'installation des réfugiés syriens. Ce secteur associatif a pour centre névralgique la ville du Six Octobre. II est alors occupé par divers réseaux de militants, activistes, travailleurs sociaux,

8. BANEGAS, Richard, POMMEROLLE, Marie-Emmanuelle, SIMEANT, Johanna, «Lutter dans les Afriques, Genèses, avril 2010, n81, p. 2-4.

9. BAYART, Jean-François, L'État en Afrique. La politique du ventre, Paris, Fayard, 1989. Mais aussi avec: TOULABOR, Comi, MBEMBE, Joseph-Achille, La politique par le bas en Afrique noire: contribution à une problématique de la démocratie, Paris, Khartala, 1992. 
volontaires ou encore hommes d'affaires et artistes. Pour comprendre les pratiques de ces acteurs, il est primordial de resituer leur action dans une lutte contre le désengagement et la démobilisation. Ces derniers pourraient être provoqués, selon eux, par la migration forcée qui les déconnecte physiquement du contexte syrien et les ampute d'une partie considérable de leur capacité à agir contre le régime de Bachar al-Assad. Dans les discours recueillis au cours des enquêtes de terrain, le sentiment d'inutilité et de dépossession de la lutte politique, une fois arrivés en Égypte, est très fort. L'action sociale correspond ainsi à une reconversion dans le mouvement migratoire d'un engagement politique antérieur. C'est le cas de Khaïs, qui fonde I'association Zaytoun au printemps 2012. Khaïs vivait aux Emirats Arabes Unis jusqu'en février 2012. C'est à ce moment qu'il est expulsé du pays, pour y avoir organisé des manifestations contre le régime syrien. Druze originaire de Sweida, Khaïs vit dans le quartier de Dokki, au Caire.

Lorsqu'il arrive en Égypte, un ami syrien, vivant aussi aux Emirats Arabes Unis, I'a mis en relation avec un ancien collègue et ami égyptien. Ce dernier met à sa disposition un appartement inoccupé à Dokki, dont il est propriétaire. Très vite, Khaïs, qui a la charge du lieu, y accueille un nombre important d'opposants syriens, tous juste arrivés et ne disposant pas d'autres endroits où aller. L'appartement se transforme en un îlot révolutionnaire, où des Syriens de milieux et d'univers très variés échangent sur le mouvement de contestation. Particulièrement actif à son arrivée, Khaïs souhaite maintenir son engagement et poursuivre les mobilisations entamées à Abu Dhabi et Dubaï. Pour lui et ses amis, les Syriens réfugiés en Égypte sont avant tout des victimes de Bachar al-Assad, "leur venir en aide c'est donc continuer à lutter contre le régime ${ }^{10}$. Il se met rapidement en relation avec des personnalités politiques syriennes œuvrant également pour les réfugiés syriens, ainsi qu'avec certains amis désireux eux-aussi d'agir, et il pense ses premières actions.

Ensemble, ils organisent des évènements culturels destinés à collecter des dons et à offrir une "zone de repli » ${ }^{11}$ aux revendications, aux discours et aux mobilisations qui ne peuvent plus avoir lieu directement en Syrie. Ils se lancent notamment dans la réalisation de festivals et concerts, dans des lieux touristiques alors désertés par leur population habituelle. Ils parviennent par exemple à réunir des vedettes syriennes, en exil dans différents pays. Ingénieur, informaticien, chargé de développement de formation, lui et ses amis mettent au service de l'action caritative et sociale leurs compétences professionnelles, réactivant ainsi un capital culturel et scolaire mis à mal par la migration forcée. Les actions qu'ils mènent leur permettent en parallèle de

10. Entretien réalisé le 18 janvier 2014, avec Khaïs, fondateur de I'association Zaytoun.

11. Entretien réalisé le 18 janvier 2014, avec Khaïs, fondateur de l'association Zaytoun. 
s'intégrer dans des projets transnationaux de soutien au mouvement révolutionnaire syrien. Ils y restructurent un capital social, des réseaux propres à leur nouvelle situation, qui faciliteront plus tard leur démarche pour quitter I'Égypte $^{12}$. Ces diverses actions visent à récolter des fonds, qu'ils réinvestissent dans l'aide aux réfugiés syriens en Égypte. La plupart d'entre eux bénéficient $d^{\prime}$ un capital économique qui leur permet de ne pas attendre de rémunérations de ces activités, d'autres ont déjà trouvé un emploi, dans des ONG locales pour la majorité.

En quelques mois, motivé par le succès de ses premières actions, Khaïs décide de fonder l'association Zaytoun, afin d'avoir une structure où développer ses projets. Cette dernière est très présente dans les entretiens réalisés auprès de réfugiés de la ville du Six Octobre. Zaytoun a joué un rôle important en termes d'accompagnement de ces derniers durant la période Morsi. Grâce à leur réseau de connaissances et à leur inventivité, les volontaires de Zaytoun organisent en particulier des journées dans des parcs d'attraction égyptiens, gratuites pour plus de trois cent enfants. Ils arrivent à convaincre des partenaires et donateurs syriens et égyptiens de mettre à disposition leurs infrastructures (écoles privées, parcs d'attraction, locaux professionnels ou privés) pour y accueillir des réfugiés. En parallèle, les levées de fonds menées auprès de riches hommes d'affaire et artistes syriens, financent le reste des activités: organisation d'ateliers et journées récréatives pour jeunes enfants, distribution de biens de première nécessité.

Soucieux du désœuvrement dans lequel se trouvent les adolescents syriens déscolarisés, ils créent aussi des ateliers plus spécifiques dans un autre appartement mis aussi à leur disposition, situé dans le quartier de Saad Zaghoul, au centre-ville du Caire. Ils souhaitent ainsi amoindrir les effets négatifs de l'exil sur la jeunesse syrienne, en leur apportant des connaissances variées, en langues étrangères, en maîtrise des outils informatiques, ainsi qu'un lieu qui leur évite de "traîner dans la rue comme les Égyptiens " ${ }^{13}$. Mais le but est aussi de leur offrir un espace de discussion sur la Syrie, sa "révolution». Zaytoun met en place des groupes de parole sur comment en parler et témoigner, pour continuer à être mobilisés même de loin. Les animateurs de ces échanges veulent aussi transmettre des connaissances historiques, culturelles afin que ces jeunes syriens «ne perdent pas leur identité en Égypte ${ }^{14}$. Le retour en Syrie est ainsi omniprésent et envisagé collectivement. Chacun est encouragé

12. Les réseaux militants que Khaïs rejoint l'informent par exemple sur les meilleurs moyens de demander I'asile en Europe: qui contacter, à quel moment, quels pays préférer au regard des orientations politiques et cadres législatifs, comment accomplir les démarches, pour quels coûts etc.

13. Entretien réalisé le 18 janvier 2014, avec Khaïs, fondateur de I'association Zaytoun.

14. Entretien réalisé le 18 janvier 2014, avec Khaïs, fondateur de I'association Zaytoun. 
à développer des compétences professionnelles et politiques qui pourraient être utiles à la reconstruction du pays, après la fin de ce qui s'apparente de plus en plus à une véritable guerre.

\section{Un contexte politique égyptien propice au secteur associatif}

En parallèle des initiatives lancées par les acteurs syriens, on note une quasi-absence de relais étatiques au Six Octobre. Leur présence serait pourtant le gage de la mise en œuvre d'une politique publique, allant dans le sens des discours du président Mohamed Morsi à partir de son élection en juin 2012. Le secteur associatif de l'accompagnement aux réfugiés syriens fonctionne donc dans un environnement caractérisé par un fort niveau d'autonomisation vis-à-vis de l'État d'accueil. On émet l'hypothèse que cette autonomisation est le fruit d'un relâchement de l'appareil sécuritaire entre 2011 et 2013. Or cet appareil sécuritaire vise communément au contrôle des associations locales telles que Zaytoun. II illustre par ailleurs plus largement une instabilité politique nouvelle au sein des organes étatiques, elle-même due en partie au renouvellement du personnel politique avec I'arrivée au pouvoir des Frères musulmans ${ }^{15}$. La politique de décharge, évoquée en introduction, ne serait pas simplement coproduite par les acteurs du secteur associatif et les appareils étatiques. Le climat d'instabilité politique entraînerait plutôt le relâchement de l'appareil sécuritaire ainsi que des divergences d'orientations politiques suivies par les acteurs de l'État. Ce dernier point crée des opportunités et des fenêtres d'action pour les acteurs qui veulent se mobiliser.

Questionner les acteurs impliqués sur leur capacité à pérenniser leurs actions entre 2011 et 2013 s'est révélé être une démarche pertinente à la fois pour comprendre leurs stratégies et pour saisir la gestion par l'État égyptien de ce nouveau secteur associatif. Khaïs affirme par exemple avoir été dans l'impossibilité de poursuivre ses activités après le coup d'État militaire de juillet 2013. Deux raisons I'expliquent selon lui. Premièrement, les financements disparaissent après juillet 2013, en raison de la répression contre les Frères musulmans, les Syriens étant alors stigmatisés comme soutien de ces derniers. Deuxièmement, les pressions de la Sûreté nationale égyptienne sur les Syriens augmentent et les contraignent à revoir coûts et risques pris dans I'engagement. Par effet de comparaison, l'après 2013 permet de comprendre plusieurs choses sur la période 2011-2013 qui nous intéresse ici.

15. Pour plus de détails, voir: ROUGIER, Bernard, LACROIX, Stéphane (Dir.), L'Égypte en révolutions, PUF, 2015. 
Tout d'abord, le ministère des affaires sociales, connu ${ }^{16}$ pour être un appareil de contrôle du secteur associatif, est toujours à ce moment en capacité de limiter les enregistrements d'associations. En effet, malgré une forte implantation et une visibilité dans l'espace public au Six Octobre, des moyens financiers et une équipe de volontaires active, I'absence de soutien auprès de I'administration locale ou de notabilités politiques égyptiennes empêche Khaïs d'enregistrer son association. Des soutiens fréristes auraient pu aider à contrebalancer cette situation, mais Khaïs et ses collègues n'en bénéficient pas. Cette situation est courante en Égypte, où d'autres associations, telles que I'association AMERA offrant une assistance juridique aux réfugiés, restent des années durant la période Moubarak sans enregistrement auprès du ministère des affaires sociales. Néanmoins, dans une période d'incertitude et d'instabilité politique, les risques encourus sont différents et dans les perceptions des acteurs en question, cette officialisation de leur action aurait pu être déterminante pour résister à la crise de l'été 2013. L'association AMERA justement $\mathrm{n}^{\prime} \mathrm{y}$ résiste pas, et est contrainte de fermer en 2014 en raison de cette absence d'enregistrement légal. En paralysant les procédures d'enregistrement des associations, le ministère des affaires sociales les cantonne ainsi dans le domaine de l'informel, propice à une gestion arbitraire des institutions de ce type (fermeture injustifiée, accès aux données, menace sur les membres, racket et corruption).

Dans le même temps, et de manière paradoxale, l'appareil sécuritaire n'intervient pas durant les actions menées par Zaytoun avant l'été 2013. Le dispositif, qui encadre strictement les manifestations publiques via un système d'autorisations à obtenir auprès de la Sûreté nationale avant chaque évènement, ne fonctionne que de manière très aléatoire et éparse entre 2011 et 2013. L'espace public au Six Octobre est en effet occupé entre 2011 et 2013 d'une manière inédite pour le contexte égyptien, avec une multitude d'évènements s'y déroulant, sans restriction ni encadrement notable. L'appareil sécuritaire et policier au cœur de l'État égyptien, bien qu'il résiste à la crise politique ouverte par la chute de Moubarak, perd donc de sa capacité à administrer le local. Cette situation contraste particulièrement avec l'après coup d'État de juillet 2013, où l'une des premières mesures prises vise à interdire les manifestations dans l'espace public.

La chute de Moubarak et la victoire des Frères musulmans aux élections créent donc une compétition pour le pouvoir au sein des organes de l'État, visible aux éléments contradictoires dans la gestion du secteur associatif syrien. Les bouleversements qui en découlent ont des conséquences directes

16. Sur ce sujet, voir: BEN NAFISSA, Sarah, "Le financement des services sociaux du secteur associatif à référent islamique en Égypte», Égypte/Monde arabe, Première série, 12-13 | 1993, mis en ligne le 08 juillet 2008, consulté le 03 juillet 2016. URL: http://ema.revues.org/1270 
sur les modalités de l'action sociale. Ils remettent en effet en cause les arrangements routiniers que les institutions administratives et sécuritaires entretiennent avec la population. Comme le rappelle Johanna Siméant ${ }^{17}$, il importe en effet de penser le conflit non pas seulement «là où on s'attend à le trouver (dans «le» mouvement social)» mais également en prenant en compte «l'activité des autorités, ce qui invite à considérer ces dernières non seulement dans leur rôle répressif mais aussi dans leur rôle de pourvoyeuses. » Les autorités sont en particulier pourvoyeuses de ces arrangements, qui peuvent prendre des formes très variées (mécanismes de cooptation, pratiques clientélistes, etc.) et qui, dans cette situation non-routinière, sont remis en cause et sujets à d'importantes renégociations. C'est dans ce cadre qu'il faut penser les marges de manœuvre, les modalités et les formes de l'action que les associations syriennes tentent de négocier.

L'État égyptien dessine les contours des modalités de l'action sociale, de I'engagement politique, en contraignant les acteurs à composer avec lui et le réel social qu'il implique ${ }^{18}$. Ainsi, même en période de relâchement sécuritaire et d'instabilité institutionnelle, l'action sociale se construit en relation avec les champs du pouvoir. On constate au cours de cette période que l'État reste pourvoyeur d'arrangements, mais de façon plus hasardeuse, parfois paradoxale. On a évoqué les partenaires égyptiens, investis techniquement et financièrement dans les projets de l'association Zaytoun, les lieux de l'espace public égyptien où se tiennent les activités, les rendez-vous, réunissant parfois des centaines de Syriens, donc forcément visibles et tolérés par les autorités égyptiennes. Dans le même temps, d'autres ministères et institutions publiques, telles que le ministère de la culture, la direction de l'Opéra du Caire, I'Université du Caire via le département des Beaux-Arts, soutiennent aussi les actions menées par Zaytoun. Lors d'un premier entretien réalisé avec Khaïs, celui-ci explique que ces soutiens se font «de personne à personne, parce que tel ou tel membre du ministère aime bien Zaytoun et ce que nous (les membres de l'association) organisons, parce qu'un autre a visité la Syrie ou a une cousine mariée à un Syrien, que son père lui parlait des atrocités commises par Hafez al-Assad, il n'y a pas vraiment de règles » ${ }^{19}$.

17. SIMEANT, Johanna, «Economie morale» et protestation - quelques détours africains, Genèses 2010/4, n81, p. 142-160. Le passage cité se trouve page 151.

18. Voir par exemple: BEN NAFISSA, Sarah, "Verrouillage autoritaire et mutation générale des rapports entre l'État et la société en Égypte», Confluence Méditerranée, $\mathrm{n}^{\circ}$ 75, 2010, p. 137-150.

Ou encore: BEN NAFISSA, Sarah, «Ca suffit?», «le haut» et le «bas » du politique en Égypte»Politique Africaine, $\mathrm{n}^{\circ}$ 108, 2008, p 5-24.

19. Entretien réalisé le 18 janvier 2014, avec Khaïs, fondateur de l'association Zaytoun. 
On note donc une dissonance dans les décisions politiques prises par exemple d'un côté par le ministère des affaires sociales et de l'autre par celui de la Culture. Alors que les pressions s'intensifient après 2013, le ministère de la culture continue par exemple à être partenaire de Zaytoun pendant quelques mois, "sous le radar ${ }^{20}$, en finançant une partie des évènements artistiques organisés. On peut supposer que plusieurs tendances se confrontent: certaines institutions du gouvernement sont dirigées par des proches du président islamiste et suivent sa ligne politique, d'autres sont traditionnellement plus proches de l'appareil de sécurité et poursuivent d'autres enjeux ${ }^{21}$. Cette instabilité politique offre à Zaytoun des stratégies d'alliance et de soutien qui restent incertaines et fluctuantes, et la prive d'un réseau solide de partenaires discrets et avisés, capables de résister aux différentes secousses de la crise politique. Or, ce sont ces derniers qui permettent aux associations et structures locales de «bricoler» avec l'appareil autoritaire et ses rouages.

En parallèle, il est important de rappeler que les engagements politiques de Khaïs et de ses collègues ne sont pas dissimulés. Les membres actifs de Zaytoun sont pour la plupart proches de la Coalition syrienne, alors implantée au Caire. Ceci va là aussi contre un système qui avait pour stratégie jusqu'en 2011 la dépolitisation des institutions politiques. Cette dépolitisation imposée ne s'applique pas uniquement aux gouvernés égyptiens, elle s'applique à toute personne pouvant menacer l'ordre établi. Or, on peut supposer que ces configurations répressives n'ont pas totalement disparu, dans la mesure où l'État en lui-même n'a pas été profondément réformé. On peut aussi émettre l'hypothèse que ces configurations répressives auraient pu être atténuées si Khaïs et ses collègues avaient affiché d'autres couleurs politiques.

\section{Logiques partisanes fréristes et opposants syriens au Caire: un jeu d'alliances}

La plupart des acteurs mobilisés autour de Khaïs s'exilent en Égypte alors que le mouvement révolutionnaire syrien n'a pas encore basculé dans le conflit armé et la guerre civile. Ils ont donc en partage un univers de sens marqué par les mobilisations pacifiques contre le régime de Bachar al-Assad: «Ce qui est important pour nous $c^{\prime}$ est de défendre le soulèvement civil de cette Révolution. C'est ce en quoi nous croyons ${ }^{22}$. Lorsqu'il décrit ces mobilisations, Khaïs met l'accent sur le fait que ces dernières suivaient de près le modèle des mobilisations en Syrie: utilisation des réseaux sociaux pour

20. Extrait d'un entretien réalisé le 18 janvier 2014, avec un membre de Zaytoun.

21. Pour plus de détails, voir: ROUGIER, Bernard, LACROIX, Stéphane (Dir.), L'Égypte en révolutions, PUF, 2015.

22. Entretien réalisé le 18 janvier 2014, avec Khaïs, fondateur de I'association Zaytoun. 
$\mathrm{s}^{\prime}$ organiser et se mobiliser, mise en place de rassemblements en vue de manifestations pacifiques dans l'espace public, choix des slogans et motifs de la revendication en concertation avec ceux utilisés en Syrie, et notamment dans sa région d'origine. La politisation de Khaïs s'est donc faite hors des frontières syriennes, en suivant dans les médias et via ses proches les premières mobilisations en 2011. Son engagement politique s'est concrétisé en Égypte, où il devient un opposant politique en rejoignant dès son arrivée le Conseil National Syrien (CNS). Khaïs obtient alors via ses nouveaux réseaux militants, un emploi à mi-temps comme informaticien pour la Coalition syrienne au Caire (Coalition Nationale des Forces de l'Opposition et de la Révolution CNFOR, qu'on appellera Coalition pour plus de commodité). Il occupe ce poste jusqu'à son départ par bateau pour l'Europe en 2014. C'est aussi dans le cadre de son investissement auprès du CNS qu'il rencontre une partie des personnes avec lesquelles il fonde Zaytoun.

Si Khaïs explique son arrivée en Égypte par une «solidarité révolutionnaire ${ }^{23}$ espérée et attendue, et une fraternité révolutionnaire qui lui paraît à l'époque évidente, les désillusions, les difficultés d'intégration, le décalage de représentations et aussi d'opinions entre Syriens et Égyptiens le conduisent très vite à évoluer dans une "bulle ${ }^{24}$ syrienne. Celle-ci est pour ainsi dire déconnectée de la scène politique et sociale égyptienne. Khaïs ne rencontre que très peu de révolutionnaires égyptiens par exemple, aucun lien politique ne se met en place entre l'organisation dont il fait partie et des organisations politiques locales. II retrouve des amis syriens, arrivant comme lui des pays du Golfe ou de Syrie, et c'est avec eux qu'il vit et se mobilise. Le bureau du CNS au Caire ne dispose que de très peu de ressources et n'a donc pas les moyens d'employer du personnel. Khaïs y travaille bénévolement en dehors de son travail pour la Coalition. En contrepartie, il y tisse un réseau de contacts précieux qui lui permettent de développer et trouver des fonds pour les actions de Zaytoun. Les membres du CNS soutiennent et financent en effet en partie I'association. Ils composent la part stable et régulière des fonds levés. Khaïs devient ainsi très vite le bras droit du représentant du CNS au Caire, Jaber al-Choufi. Signataire de la Déclaration de Damas et opposant syrien de longue date, ce dernier témoigne des logiques partisanes qui expliquent le mode de fonctionnement des relations entre les opposants syriens et l'État égyptien sous Morsi. Il s'agit ici des logiques partisanes régissant l'action politique, mais les acteurs du secteur associatif rencontrés au cours de l'enquête de terrain aboutissent aux mêmes conclusions. Jaber al-Choufi explique:

23. Entretien réalisé le 18 janvier 2014, avec Khaïs, fondateur de l'association Zaytoun.

24. Entretien réalisé le 18 janvier 2014, avec Khaïs, fondateur de I'association Zaytoun. 
«A l'ouverture du bureau, nous voulions rencontrer des hommes politiques, mais nous n'obtenons pas de réponse. (...) Nous essayons d'avoir une représentation légale (du CNS) auprès du gouvernement égyptien mais c'est un échec. On en reste aux relations de personne. Le travail est limité. Les relations se créent entre Frères musulmans, les Frères musulmans syriens créent leurs propres relations avec le gouvernement égyptien ${ }^{25}$.

Khaïs complète son propos en expliquant que le bureau du CNS en Égypte «n'est pas du tout islamiste», ce qui a pu être vérifié lors d'observations durant des réunions politiques. Les membres du CNS au Caire sont majoritairement issus de la gauche sécularisée syrienne, ils prônent une séparation du religieux et du politique, ont milité clandestinement dans le passé au parti communiste syrien pour les plus anciens. Parmi les militants les plus jeunes, on constate une nette défiance vis-à-vis des mouvements islamistes, perçus comme les «voleurs de la révolution syrienne ${ }^{26}$ et la cause de l'échec de la résistance civile syrienne face au développement des mouvements djihadistes. Khaïs déclare par ailleurs: "le 30 juin, je suis descendu manifester contre les Frères. Je n'aimais pas Morsi».

Pour mieux comprendre la situation décrite par Jaber al-Choufi, il est important d'évoquer les débuts de la Coalition, avant son installation à Istanbul à l'été 2013. Elle est créée le 11 novembre 2012 à Doha. Parmi les forces importantes de l'opposition syrienne, elle réunit deux groupes: le Conseil National Syrien (CNS) et les Frères musulmans syriens. Elle est alors dirigée par Mouaz El Khatib, imam sunnite et figure importante de l'opposition islamiste modérée ${ }^{27}$, proche idéologiquement des Frères musulmans égyptiens. C'est sous sa direction et grâce à ses réseaux au sein de l'Association égyptienne que la Coalition s'installe au Caire en janvier 2013. Ces éléments de contexte permettent de comprendre les blocages que rencontrent Khaïs avec Zaytoun et qui sont le reflet d'une situation plus large:

« Notre principal problème pour développer Zaytoun est l'argent. Avant juillet 2013, personne ne cherche à travailler avec nous. Les Égyptiens cherchent plutôt à discuter avec des Frères musulmans syriens, des salafistes, qui eux ont déjà de I'argent. (...) Sous Morsi, les contacts avec les Frères musulmans syriens sont au top! Par contre, les Frères musulmans égyptiens ne cherchent à aider aucun autre groupe de l'opposition».

25. Extrait d'un entretien réalisé avec Jaber al-Choufi, le 23 janvier 2014, au Caire, Égypte.

26. Extrait des observations menées lors des réunions politiques du Conseil National Syrien, entre janvier et mars 2014, au Caire, Égypte.

27. PIERRET, Thomas, Baas et Islam en Syrie, Presses Universitaires de France, 2011, p. 174. 
Or action sociale et action politique étant intimement liées, I'exclusion des logiques partisanes - qui semblent être le seul vecteur possible de contact avec les autorités égyptiennes entre 2012 et 2013, provoquent des impasses pour les acteurs syriens mobilisés auprès des réfugiés.

En conclusion, les mécanismes de la coproduction de la politique de décharge, visant à accompagner l'arrivée et l'installation des réfugiés syriens, sont complexifiés par un contexte d'instabilité politique. Cette instabilité politique transpose, au sein de l'État lui-même, la polarisation politique autour de l'accès au pouvoir des Frères musulmans. Le soutien de Morsi aux réfugiés syriens à une échelle nationale, combiné à des dissonances politiques au sein des organes étatiques et à des décisions et des orientations parfois contradictoires à l'échelle des institutions, constituent une brèche dans un système jusqu'alors verrouillé. Les acteurs du secteur associatif I'exploitent pour mettre en œuvre sur le terrain les mesures que Morsi promet, mais que l'État égyptien relaie très sporadiquement. La politique de décharge s'opère donc dans le cadre de stratégies d'alliances vertueuses avec les Frères musulmans, hypothèse que l'on développera ultérieurement. En cas d'exclusion des logiques partisanes fréristes, la brèche ouverte offre un espace de tolérance - certes inédit - pour mener des actions, mais avec des moyens limités qui ne permettent pas d'assurer la pérennité de l'aide sociale apportée aux réfugiés.

\section{LA MOBILISATION DES ACTEURS ASSOCIATIFS ET DES MOUVEMENTS ISLAMISTES EN FAVEUR DES RÉFUGIÉS SYRIENS}

Les techniques de mobilisation fréristes au service de la prise en charge des réfugiés

La première partie de cet article a montré que l'autonomisation du secteur associatif prenait racine dans la combinaison entre un relâchement autoritaire et une instabilité politique au sein des appareils étatiques. Ce climat est davantage propice aux alliances interpersonnelles et aux logiques partisanes qu'aux réformes profondes de l'État, de ses pratiques et de son personnel. J'émets l'hypothèse que cette autonomisation vis-à-vis de l'État s'appuie sur l'impulsion donnée par les Frères musulmans et leurs dispositifs de prise en charge des questions sociales. Marie Vannetzel explique en effet: 
Les Frères se soustraient ainsi partiellement aux pressions pesant sur le système clientéliste global, grâces à des dispositifs pratiques ${ }^{28}$ permettant de traiter la demande sociale de service à moindre coût. Surtout ces dispositifs, en mettant en jeu des espaces plus ou moins informels, insérés dans le tissu social local, s'avèrent particulièrement propices à la construction des notabilités et à l'entretien quotidien des réseaux. ${ }^{29}$

En ce sens, cette hypothèse nous conduit à en formuler une suivante: les Frères musulmans opèrent durant la présidence de Morsi une reconversion des pratiques acquises dans la semi-clandestinité ${ }^{30}$ pour gérer et administrer I'arrivée et l'installation des réfugiés syriens. Leur faible ancrage institutionnel au sein des appareils d'État ne leur permet pas de mettre en œuvre une véritable politique publique. Ils continuent donc d'agir dans les espaces laissés vacants par l'État, en raison du mode de gouvernance par décharge qui prévaut. Ils investissent ainsi l'informel et l'administration du local. Cette politique de décharge est co-produite par les acteurs du secteur associatif et les Frères musulmans alors au pouvoir.

Le cas de I'association Unique illustre bien ces réflexions. L'association Unique est fondée par plusieurs Égyptiens impliqués localement au Six Octobre et mobilisés auprès des réfugiés syriens. Myriam est l'une des fondatrices de ce projet et elle en est aussi la directrice générale. Âgée d'environ quarante cinq ans, elle est née au Koweït d'un père égyptien et d'une mère syrienne ayant la nationalité canadienne. Elle grandit au Canada, à Toronto, où elle poursuit des études en économie et statistiques. Myriam se marie à un avocat égyptien, avec qui elle a six enfants. Elle se décrit comme une home-schooling mother (une mère assurant l'éducation de ses enfants à la maison, sans avoir recours à une scolarisation classique). Le couple décide de revenir vivre en Égypte en 2000. Elle vit ainsi au Six Octobre depuis plusieurs années au moment du déclenchement du mouvement révolutionnaire de 2011. Elle explique qu'après ce dernier, elle ressent un fort besoin de s'engager. Par ailleurs, l'arrivée de réfugiés syriens la touche, ce pays étant celui de sa mère. Elle commence à se mobiliser alors qu'elle déménage pour une nouvelle maison dans la ville du Six Octobre. Elle souhaite faire don de certains biens. En raison de ses origines syriennes, Myriam connaît ce dialecte, arrive facilement à échanger avec les réfugiés et à rencontrer les différents groupes

28. VANNETZEL, Marie, "Les voies silencieuses de la contestation: les Frères musulmans égyptiens, entre clientélisme et citoyenneté alternative», Presses de Sciences $P_{O}, 2008$, p.31. Il s'agit $\mathrm{d}^{\prime}$ " associations locales de bienfaisance, syndicats et ordre professionnels, universités, conseils de justice populaire».

29. Idem.

30. VANNETZEL, Marie, Les Frères musulmans égyptiens, enquête sur un secret public, Khartala, 2015. 
qu'ils constituent dans la ville. Elle déclare être entrée en contact avec les premiers réfugiés via une organisation qu'elle qualifie d'ONG caritative, et qu'elle nomme: Gamấa Islâmiyya. Elle explique que son aide a été "décisive» à sa socialisation dans ce nouvel univers: "L'un des acteurs principaux (de l'aide aux syriens) est la Gamâ'a Islâmiyya. Cette association commence par trouver des logements aux Syriens à Six Octobre, elle paie les loyers, fournit l'équipement ménager, du lait, de la nourriture, des couvertures, à tel point que le nombre de Syriens à faire appel à elle explose » ${ }^{31}$.

La Gamâ'a Islâmiyya qu'elle évoque n'est pas sans rappeler l'organisation salafiste égyptienne du même nom. Au cours de l'enquête de terrain, réalisée entre 2013 et 2014 et marquée par un fort de climat de suspicion, lié à la répression violente des mouvements islamistes par le nouveau régime d'Abdel Fattah al-Sissi, Myriam refuse catégoriquement de préciser de quel acteur il s'agit. Elle s'oppose aussi fermement à organiser une rencontre ou une mise en contact avec la Gamấa Islâmiyya dont elle parle. Elle déclare qu'il est désormais «impossible de les rencontrer», et ajoute: "ils ne partagent pas les mêmes valeurs, ils refuseront de vous parler, surtout en ce moment ${ }^{32}$. Il y a une nette contradiction dans son propos entre la proximité apparente qu'elle entretient avec cette organisation sous la présidence de Morsi, et sa volonté au moment de l'enquête de s'en démarquer. Les recherches effectuées ont montré qu'aucune autre association locale, enregistrée ou non, syrienne ou égyptienne, n'existait sous ce nom dans la ville du Six Octobre. De plus, d'autres entretiens réalisés ont confirmé que de nombreuses actions de solidarité et d'aide sociale étaient menées par des militants et sympathisants se revendiquant de l'organisation salafiste, Gamâ'a Islâmiyya. II est difficile de dire s'il s'agissait de l'organisation elle-même, reformée dans l'après 2011, ou d'une initiative spécifique menée par une partie de sa base militante et communément appelée par les acteurs sur le terrain Gamâ'a Islâmiyya. On parle donc de Gamâ'a Islâmiyya ici pour qualifier I'organisation elle-même, et de Gamâ'a Islâmiyya lorsqu'on évoque la notion endogène des acteurs sur le terrain.

En conflit avec les Frères musulmans dès les années 1980, la Gamâ'a Islâmiyya a néanmoins emprunté à ces derniers leur technique de mobilisation et leur est proche d'un point de vue organisationnel, grâce à un modèle aussi efficace à l'échelle locale notamment. Dans le cadre de la campagne présidentielle et de son investiture, Morsi a tout intérêt à un rapprochement avec les militants et sympathisants encore actifs de la Gamâ'a Islâmiyya. II est nécessaire de se garder d'un amalgame entre la Gamâ'a Islâmiyya et les Frères

31. Entretien réalisé le 8 janvier 2014, auprès de Myriam, présidente de l'association Unique d'aide aux réfugiés syriens.

32. Entretien réalisé le 8 janvier 2014, auprès de Myriam, présidente de l'association Unique d'aide aux réfugiés syriens. 
musulmans. Néanmoins, I'implication de militants de la Gamâ'a, dans la prise en charge des réfugiés syriens au Six Octobre, est intéressante car elle se fait en partenariat avec les militants et sympathisants fréristes, et selon des modes d'action similaires.

Ces deux acteurs jouent en effet dans la ville du Six Octobre un rôle crucial. Entre l'Égypte, le Liban - d'où la majorité des Syriens prend I'avion pour Le Caire, et la Syrie, les réfugiés syriens se transmettent des contacts parmi les réseaux fréristes ou de la Gamâ'a Islâmiyya. Ainsi, lorsqu'ils arrivent à I'aéroport du Caire, bien souvent après avoir dépensé tout leur argent dans la sortie de Syrie et les billets d'avion, ils sont déjà renseignés, ou apprennent par le bouche à oreille sur place, vers quels acteurs se tourner. Dans la plupart des cas, on leur indique une mosquée au Six Octobre où ils se rendent directement. Les acteurs Frères musulmans et proches de la Gamâ'a Islâmiyya sont très vite dépassés le nombre de Syriens à aider et s'organisent en conséquence. C'est par exemple la Gamấa Islâmiyya qui va choisir où installer les Syriens les plus pauvres et ne disposant pas des ressources nécessaires pour prendre en charge leur installation. Elle choisit ainsi les faubourgs non-planifiés d'un quartier appelé Masakan Othman, à la frontière avec le désert qui borde la ville du Six Octobre. Les immeubles de ce quartier ont été construits à la suite d'un accident pétrochimique survenu en périphérie du Caire également. Cet accident avait nécessité de déplacer des familles égyptiennes dont les habitations avaient été détruites. Les loyers y sont très bas, ce qui explique le choix de la Gamấa Islâmiyya, qui peut ainsi venir en aide à un nombre plus important de familles. Mais très vite, les incidents se multiplient entre Égyptiens et Syriens, les habitants égyptiens reprochant aux Syriens de bénéficier d'une aide sociale qui devraient selon eux leur revenir. Des cas d'agressions et de viols de femmes et d'enfants syriens par des membres de gangs égyptiens du quartier sont recensés. En parallèle, les réfugiés syriens doivent au quotidien négocier leur présence auprès de groupes de trafiquants et des réseaux de criminalité qui régissent cet espace. Ils décident ainsi d'organiser des comités locaux pour assurer leur sécurité, reprenant le modèle les tansiqiat syriens (comités populaires nés du mouvement révolutionnaire et visant à assurer la sécurité des civils et des manifestants).

La Gamâ'a Islâmiyya décide progressivement début 2013, de déplacer les réfugiés syriens dans un autre quartier de Six Octobre, situé à un ou deux kilomètres de Masakan Othman, de I'autre côté de l'axe routier qui coupe ce ghetto du reste de la ville. Après la chute de Morsi en juillet 2013, c'est l'association Unique qui prend à sa charge les derniers déménagements, la Gamấa Islâmiyya n'étant plus en mesure d'agir. Myriam explique que les membres de la Gamâ'a Islâmiyya subissent la répression du régime et ont été emprisonnés, ont fui ou rejoint la clandestinité. L'association Unique, qui a pu se développer entre 2012 et 2013, s'occupe donc de transférer les dernières familles dans de nouveaux appartements, d'assurer leur installation, et saisit cette opportunité pour inciter les réfugiés à faire bénéficier leurs enfants des 
activités de I'association, leur assurant ainsi un cadre sécurisé et un complément à une scolarisation irrégulière ou inexistante. Ainsi, le contexte de publicisation des actions menées par des organisations islamistes, sous Morsi, révèle comment les mouvements islamistes, en collaboration avec les Frères musulmans au pouvoir, pallient le désengagement social de l'État en suivant les méthodes acquises durant les années de semi-clandestinité. Le secteur associatif et ses acteurs sont ainsi mobilisés dans ce qui devient un mode $\mathrm{d}^{\prime}$ administration du local et de la question sociale par décharge. Ce sont en effet, ces acteurs, et non pas l'État égyptien en tant que tel, qui pensent, organisent et administrent une partie importante de la population de réfugiés syriens, qui arrivent alors dans la ville du Six Octobre. Le paradoxe de cette période 2012-2013 est une coproduction de la décharge ancrée dans des pratiques nées d'une résistance à une répression des mouvements islamistes, supposée ne plus exister avec la prise de pouvoir des Frères musulmans et leur victoire aux diverses élections depuis 2011.

\section{La nécessité de «travailler sous le radar » ${ }^{33}$}

Lors d'entretiens, réalisés après l'été 2013, avec d'autres acteurs du secteur associatif de l'aide aux réfugiés syriens, mais aussi du secteur associatif égyptien, des ONG et des bailleurs internationaux, l'appartenance supposée de Myriam aux Frères musulmans revient à plusieurs reprises. Elle apparaît sous forme de confidences chuchotées, souvent suivies d'un: "mais elle fait du bon travail, alors on fait comme si on ne savait pas... ${ }^{34}$. Cet accord implicite entre les différents acteurs rappelle le phénomène de coopération tacite décrit par Marie Vannetzel, autour du «secret public» que constituent les Frères musulmans ${ }^{35}$. Les modalités de l'action sociale décrites par Myriam pour évoquer ses débuts aux côtés des acteurs de la Gamâ'a Islâmiyya (porte à porte, évaluation des besoins, travail aux côtés des membres du Syndicat de Médecins de Six Octobre proche des Frères musulmans, absence de liens personnels entre ces acteurs) coïncident aussi avec le répertoire d'action et les techniques d'implantation des Frères musulmans. Sa trajectoire, ses discours, son habitus, rappellent la construction par l'association frériste d'un profil typique de Frères «non connu», «militant intermédiaire, ni novice (il doit être

33. Entretien réalisé le 8 janvier 2014, auprès de Myriam, présidente de l'association Unique d'aide aux réfugiés syriens.

34. Extrait d'une discussion informelle avec une notabilité locale syrienne, janvier 2014, Le Caire, Égypte.

35. VANNETZEL, Marie, Les Frères musulmans égyptiens, enquête sur un secret public, Khartala, 2015. 
capable d'assumer cette charge), ni confirmé (sinon il serait connu des services de sécurité): et sa mission est entre autres de veiller à le rester en se livrant avec prudence à ses activités $»^{36}$.

Plus que savoir si Myriam fait partie des Frères musulmans ou non, ces éléments témoignent du maintien après 2011, et plus particulièrement sous Morsi, de modes d'organisation de l'action politique et sociale hérités des années de semi-clandestinité. Les organisations islamistes ont subi de plein fouet la répression des régimes précédant 2011, et ils ont ainsi développé des savoirs et des dispositifs qui leur permettent de contourner le contrôle autoritaire des appareils étatiques. Myriam et ses collaborateurs ont transféré cette expérience au sein de leur projet associatif. Quelques mois après avoir rejoint la Gamấa Islâmiyya, en avril 2012, Myriam est intégrée à un groupe de bénévoles, qui ne se connaissent pas encore et que l'organisation a constitué elle-même. Myriam se lance dans la création d'une base de données avec tous les contacts de Syriens qu'elle rencontre depuis 2011 et le recensement de leurs besoins. Face à l'étendue des besoins qu'elle constate, l'idée naît de monter une association, idée qu'elle développe avec les autres personnes de son groupe dès avril 2012, avant de déposer les statuts de I'association Unique en août 2012. Ils sont donc six médecins et six citoyens venant d'horizons différents. Dans ce projet, plusieurs types de socialisation et de capitaux se mêlent. Les fondateurs mélangent ainsi à la fois les stratégies de contournement du contrôle de l'action sociale, héritées de leurs actions dans des organisations islamistes réprimées pendant de longues années, et des stratégies davantage liées à leurs expériences professionnelles et personnelles à l'international (en tant que médecin, avocat ou mère de famille assurant elle-même l'éducation scolaire de ses enfants dans le cas de Myriam). Ce dernier point leur permet de s'attirer les faveurs du champ des ONG et des institutions internationales.

Myriam se familiarise rapidement aux éléments de langage du secteur du développement et de l'action humanitaire, en raison de sa maîtrise parfaite de I'anglais et des années passées à étudier dans une université canadienne. Comme énoncé précédemment, lorsqu'elle parle de la Gamấa Islâmiyya, Myriam prend le soin de ne pas parler d'une organisation islamiste locale, mais d'une ONG - elle emploie le diminutif anglais «NGO». Elle insiste par ailleurs sur le caractère apolitique de son association Unique à plusieurs reprises: "Unique fait de la charité et surtout pas dıaction politique. Nous sommes une ONG, nous n'avons pas diengagement politique, nous travaillons avec tout le monde, avec des ONG religieuses et des ONG internationales » ${ }^{37}$.

36. VANNETZEL, Marie, "Secret public, réseaux sociaux et morale politique. Les Frères musulmans et la société égyptienne», Politix, n²2, 2010, p. 92.

37. Entretien réalisé le 8 janvier 2014, auprès de Myriam, présidente de l'association Unique d'aide aux réfugiés syriens. 
Le label «ONG» est couramment utilisé pour décrire les partenaires du champ. Myriam et ses collaborateurs savent que le contrôle par les appareils étatiques du secteur associatif a pour vocation la dépolitisation du fait social. Ils maîtrisent donc parfaitement l'injonction à l'apolitisme, omniprésente en situation de contrainte ${ }^{38}$. Parler d'organisations non-gouvernementales, c'est donc à la fois s'ancrer dans cet "à côté » du politique et dans un vocabulaire international qui tend à extraire du local et de ses contraintes.

Cette maîtrise des dispositifs de contrôle de l'action sociale par l'État égyptien leur permet d'éviter les restrictions mises en place par le ministère des affaires sociales. L'association Unique n'est pas une ONG, mais une initiative locale, briguant au statut d'association à partir d'août 2012, dans le cadre législatif égyptien. Or, elle est spécialisée dans I'aide aux réfugiés syriens. La législation ne permet pas à une association d'œuvrer pour un public n'incluant pas des Égyptiens ${ }^{39}$. Pour pouvoir être enregistrée, I'association change donc sur le papier son public bénéficiaire. Aux yeux du ministère des affaires sociales qui l'a enregistrée, elle œuvre en faveur des enfants défavorisés. Ceci est possible grâce à une véritable ONG internationale, qui accepte de «faire écran» pour citer Myriam, en accueillant les projets de l'association dans ses locaux, comme destinés à un public mixte d'enfants, alors qu'elle sait que seuls des Syriens seront présents. Ici, ce ne sont pas les dispositifs issus des organisations islamistes qui leur inspirent ces démarches. Les fondateurs d'Unique perçoivent l'enregistrement, et donc I'institutionnalisation de leur association, comme une nécessité. Ils se démarquent de la majorité des organisations du secteur associatif syrien, telles que Zaytoun, qui n'entreprennent pas ses démarches et les jugent même obsolètes. On retrouve davantage ici les traces des expériences de certains membres d'Unique dans des pays étrangers et institutions internationales. Ils visent par exemple à pouvoir lever des fonds officiellement, obtenir des subventions des bailleurs internationaux avec qui ils communiquent régulièrement et aisément. Ils savent ainsi que cela est plus compliqué sans cet enregistrement. Là où les autres structures évitent l'enregistrement pour rester hors du contrôle et des restrictions sévères imposés par le ministère des affaires sociales, les

38. Sur cette question de I'apolitisme, voir: POMMEROLLE, Marie-Emmanuelle, «Militer pour et réformer par les droits de I'Homme dans les Afriques: modèles d'émancipation et modes de reproduction de l'ordre politique», Politique des modèles en Afrique - Simulation, dépolitisation et appropriation, Paris, Karthala, 2009, p. 153-170.

39. Voir aussi sur ce sujet: BEN NAFISSA, Sarah, "Le financement des services sociaux du secteur associatif à référent islamique en Égypte», Égypte/Monde arabe, Première série, 12-13 | 1993, mis en ligne le 08 juillet 2008, consulté le 03 juillet 2016. URL: http://ema.revues.org/1270 
fondateurs de l'association Unique voient dans cet enregistrement un gage de pérennisation de leurs actions et un passeport pour des partenariats avec des institutions internationales, disposant de davantage de moyens.

Pour mener à bien leur projet, ils bénéficient de l'appui d'avocats dans leur entourage et dans leur équipe, ces derniers leur apportant leur connaissance du cadre légal égyptien pour faire aboutir avec succès une procédure administrative et légale. Ainsi, en mars 2013, avant la destitution de Morsi et après de nombreuses et laborieuses démarches, ils obtiennent la licence du ministère des affaires sociales. Ceci est un fait notable au regard du très fort contrôle, évoqué en première partie, qu'exercent les régimes successifs sur la création d'associations. L'association Unique fait partie des deux seules associations locales dans le champ de l'aide aux réfugiés syriens, nées entre 2011 et 2013, à être parvenues à s'enregistrer. Or, on a vu en première partie que malgré l'élection de Morsi, le ministère des affaires sociales continuait à limiter l'accès à l'enregistrement pour les associations locales. Dans les deux cas d'associations officialisées, I'enregistrement a eu lieu au cours de la présidence de Morsi: plus aucune association de ce champ n'a rencontré de succès dans cette démarche depuis 2013. Ceci tend à confirmer l'hypothèse, développée avec le cas de Zaytoun, d'une instabilité au sein des institutions, créant des opportunités aléatoires pour les acteurs.

Si les réseaux fréristes participent donc à la structuration et à l'émergence de l'action sociale en faveur des réfugiés syriens, ils n'incitent pas pour autant ces acteurs à enregistrer leurs associations entre 2012 et 2013, car l'institutionnalisation n'est pas une pratique émanant directement des dispositifs qu'ils mettent en place pour répondre à la demande sociale. Ceci tend à confirmer l'hypothèse $d^{\prime}$ 'un faible niveau d'ancrage institutionnel des Frères durant la présidence de Morsi, en particulier au sein des institutions étatiques. Dans une forme de torsion singulière, et alors qu'ils ne subissent plus la répression du régime qu'ils incarnent désormais, les Frères musulmans égyptiens entre 2012 et 2013 paraissent encourager la continuité de stratégies de contournement des appareils étatiques.

\section{Des stratégies de contournement des appareils étatiques : un paradoxe sous Morsi?}

Après la destitution de Mohamed Morsi en juillet 2013, seules quelquesunes des institutions rassemblées sous l'égide de la société civile syrienne sont encore actives ${ }^{40}$. Qu'ont-elles bâti lors de la période précédente qui les ait rendues pérennes dans un moment de crise et de répression particulièrement violent? Le cas de I'association égyptienne Unique a montré comment une

40. D'autres réapparaitront, mais bien plus tard, sous des formes nouvelles que je n'évoquerai pas dans cet article. 
institution de ce type pouvait mettre en place, dès ses débuts, des stratégies de contournement des appareils étatiques et de leur contrôle sur les actions menées. Il est en effet important de relever que ces stratégies sont paradoxales, dans la mesure où elles visent à éviter les limites et contraintes posées par l'État égyptien, tout en étant initié et soutenu par ceux supposés incarner cet État et en être à la tête à ce moment: les Frères musulmans, sous I'autorité du président élu Mohamed Morsi. Durant les entretiens réalisés à Six Octobre en 2013 et 2014, cette situation se ressent particulièrement. Lorsqu'on pose la question «Mohamed Morsi soutient les réfugiés syriens, que fait concrètement le gouvernement? Que fait l'État?», la réponse récurrente est "rien». Et lorsqu'on poursuit en demandant: "Mais alors qui s'occupe des réfugiés syriens dans la ville du Six Octobre par exemple?", la réponse à nouveau récurrente est: "Les Syriens eux-mêmes, appuyés par les Frères ».

Au cours des années de présidence de Hosni Moubarak, l'État égyptien a donc mis en place des mesures de contrôle de l'action sociale. Dans les milieux fréristes en particulier, ceci donne lieu à un déploiement de «formes de politisation discrètes mais quotidiennes ${ }^{41}$. Les modes de gestion fréristes du social durant les années de semi-clandestinité sont omniprésents dans les modes de gestion de l'arrivée des réfugiés syriens dans la ville du Six Octobre. Ainsi, si le gouvernement Morsi ne semble pas présent pour mettre en œuvre une politique publique destinée à gérer cette arrivée importante d'une population réfugiée, les militants et sympathisants de l'organisation, eux, sont très mobilisés sur le terrain. Les Frères musulmans, face à leur incapacité à accaparer pleinement les appareils d'État et face à la faiblesse de leur ancrage institutionnel, gouvernent en reproduisant de manière désormais publique et visible ce qu'ils ont appris à mettre en place dans les configurations autoritaires préalables. Les anciens dispositifs visant à répondre à la demande sociale sont mis à la disposition des réfugiés syriens, et comblent les vides d'une politique publique en réalité inexistante dans des formes officielles et étatiques. Le secteur associatif syrien a donc largement été tributaire de logiques partisanes qui lui permettaient de s'implanter localement, comme c'est le cas au Six Octobre.

41. VANNETZEL, Marie, "A l'épreuve du passage à la légalité», Égypte en Révolutions, sous la direction de Stéphane Lacroix et Bernard Rougier, PUF, 2015, p. 53. 


\section{CONCLUSION}

Questionner les conditions de l'existence et du devenir du secteur associatif consacré à l'accompagnement des réfugiés syriens, interroger les modalités et les formes de ses actions, informent sur les modes de gestion des actions politiques et sociales. Une multiplication des initiatives apparaît entre 2011 et 2013, soutenue publiquement par le président islamiste Mohamed Morsi. Elle donne à voir un contexte où les actions sociales et politiques semblent pouvoir s'exprimer dans l'espace public. Pourtant, ce sont les dispositifs informels, les stratégies d'évitement du contrôle étatique et les dynamiques partisanes qui semblent l'emporter et orchestrer les règles du jeu.

Ainsi, un contexte politique même favorable à l'intégration d'une population réfugiée, dans cette configuration politique où les Frères musulmans n'ont pas profondément et réellement réformé l'État égyptien, reste un cadre de contrainte. Cette contrainte induit des modes d'administration et de gestion de la demande sociale donnant à voir une politique de décharge paradoxale. Ce n'est plus simplement l'État égyptien qui tolère, tout en le contrôlant de près, que le secteur associatif pallie une partie de son désengagement social. C'est un parti, à la tête de cet État, qui organise la coproduction d'une politique de décharge, à défaut d'être en capacité de mettre en œuvre la politique publique que ses discours laissent espérer. En questionnant comment se forme, se développe et s'organise un secteur associatif, en s'intéressant aux "pratiques par lesquelles s'énonce le dissentiment " ${ }^{42}$ vis-à-vis d'un État que les acteurs syriens et égyptiens, concernés par la question de I'installation et de la prise en charge des réfugiés syriens, qualifient $d^{\prime}$ " absent» et dont ils critiquent la capacité à tenir ses promesses, on comprend que la coproduction d'une politique de décharge est le reflet d'une compétition pour le pouvoir au sein des appareils étatiques. Elle n'est pas uniquement une manière de gouverner et d'administrer le local. Elle peut aussi devenir le socle d'une tentative pour les Frères musulmans d'accès à la capacité même de gouverner, face à des appareils étatiques verrouillés de I'intérieur - l'élection de Mohamed Morsi n'ayant pas suffi à les déverrouiller. A la manière d'un cheval de Troie, les formes de coproduction particulières de la politique de décharge présenté dans cet article permettent aux Frères musulmans de pénétrer le champ de l'exercice du pouvoir. Ils mobilisent une base entraînée et engagée pour gérer la prise en charge des réfugiés syriens, à défaut d'être à même d'investir efficacement la tête de l'État pour mettre en place une véritable politique publique.

42. BANEGAS, Richard, POMMEROLLE, Marie-Emmanuelle, SIMEANT, Johanna, «Lutter dans les Afriques, Genèses, avril 2010, n81, p. 2-4. 
Avec la fermeture de nombreuses associations telles que Zaytoun, l'arrêt de multiples initiatives sociales et politiques, le départ de la Coalition syrienne pour Istanbul, c'est la fin d'une ère particulièrement propice pour les réfugiés syriens qui s'annonce en juillet 2013. Le retour de l'armée au pouvoir entraine un bouleversement des pratiques des acteurs du secteur associatif et des réfugiés syriens dans leur ensemble. Ceci s'explique par un changement brutal des perceptions les concernant. Une campagne de stigmatisation contre les réfugiés syriens est lancée par le régime et reprise par les médias locaux, à l'image du journaliste télévisuel Tawfiq Okasha encourageant publiquement les Égyptiens à agresser les Syriens qui n'auraient pas prouvé leur non-allégeance à Mohamed Morsi ${ }^{43}$. Le même constat se répète: "Les Égyptiens nous assimilent aux Frères musulmans, il n'est pas du tout question de révolution commune. C'est difficile de leur faire comprendre que nous ne sommes pas des islamistes, ils reprochent même à des chrétiens de l'être, même à des communistes. Ils ne comprennent rien ${ }^{44}$. Ainsi, après le coup d'État du 3 juillet 2013, enquêter sur le secteur associatif en faveur des réfugiés syriens en Égypte, amène à constater une évidence criante: «II ne reste plus rien, tout a fermé » ${ }^{45}$. Face à la répression menée par le nouveau régime, la quasi-totalité du secteur associatif qui avait émergé a dû arrêter ses actions, avant de les reprendre sous des formes encore plus informelles et publiquement dépolitisées.

43. Cf. I'article de Jasmin Fritzsche, Egypt's others, paru sur le site Sada, le 5 novembre 2013 et consulté le 16 janvier 2017, URL: http://carnegieendowment.org/ sada/2013/11/05/refugees-and-egypt-s-other/gsqu.

44. Entretien réalisé le 22 janvier 2014, auprès d'un ancien militant communiste syrien, investi dans des mouvements de société civile du Six Octobre.

45. Entretien réalisé le 18 janvier 2014, avec Khaïs, fondateur de l'association Zaytoun. 